\title{
Liver Dysfunction in TB-Diabetic and TB Non-Diabetic Patients Admitted in TB Referral Hospital in Western Cameroon
}

\author{
Sama LF ${ }^{1}$, Ali IM ${ }^{1,2}$, Noubom $\mathbf{M}^{3,4,5}$, Nganou Djinou OI ${ }^{1}$, Wam EC ${ }^{1,6}$, Bamou $\mathbf{R}^{7}$, Kuiate $\mathrm{J}^{1}$ and Tume CB ${ }^{1 *}$ \\ ${ }^{1}$ Department of Biochemistry, University of Dschang, Dschang, Cameroon \\ ${ }^{2}$ The Biotechnology Centre, University of Yaoundé I, Yaoundé, Cameroon \\ ${ }^{3}$ Department of Biomedical Science, University of Dschang, Cameroon \\ ${ }^{4}$ Centre Médical d'Arrondissement (CMA) De Baleng, Bafoussam, Cameroon \\ ${ }^{5}$ Centre De Diagnostic et De Traitement de la Tuberculose, Bafoussam, Cameroon \\ ${ }^{6}$ Department of Biology, Higher Teacher Training College (ENS) Bambili, University of Bamenda, Cameroon \\ ${ }^{7}$ Department of Animal Biology, University of Dschang, Dschang, Cameroon
}

\begin{abstract}
Background: Liver Function Tests (LFTs) are group of tests that help in diagnosis, monitoring therapy, and assessing prognosis of liver disease.

Methods: To estimate liver function tests in TB-DM and TB without diabetes, we conducted a cross-sectional study in sputum positive pulmonary TB patients in two TB management clinics in Bamenda and Bafoussam in the North West and West regions of Cameroon respectively from November 2014 to July 2015.
\end{abstract}

Results: Of the 189 who patients who were recruited in the study $11.2 \%(21 / 189)$ were TB-DM, 65.1\% (123/189) were TB without DM. The mean age of TB-DM was $41.38 \pm 14.36$ years with age found between 21 and 70 years whereas in TB without DM, the mean age was $35.76 \pm 17.64$ with minimum age being 12 years and maximum age 82 years. Of these participants, more than half presented abnormal liver function profile with $78.3 \%$ (148/189), 39.7\% (75/189), 88.36\% (167/189), and 91.54\% (173/189) presenting abnormal levels of ALP, GGT, ALAT and ASAT respectively. High levels of liver enzymes were observe between the two types of population with no significant

Conclusion: This study showed high levels of liver enzymes in TB-diabetic and TB non-diabetic patients but no significant difference was observed between the two populations. Therefore, proper follow-up during TB treatment should be mandatory.

Keywords: Diabetes mellitus; Pulmonary TB; Blood glucose; Toxicity

Abbreviations: ASAT: Aspartate Transaminase; ALP: Alkaline Phosphatase; ALT: Alanine Aminotransferase; GGT: GammaGlutamyltransferase; DM: Diabets Mellitus; OGTT: Oral Glucose Tolerant Test; WHO: World Health Organisation

\section{Introduction}

Liver plays a major role in metabolism and has a number of functions in the body, including glycogen storage which is made from sugars, helping to process fats and proteins from digested food, making proteins that are essential for blood to clot, decomposition of red blood cells, hormone production and detoxification [1]. Tuberculosis (TB) continues to be the leading killer among bacterial diseases worldwide $[2,3]$. It is estimated that one-third of the world's population have TB infection, and there are 9.4 million new cases of TB per year [4]. The World Health Organization (WHO) suspects that TB control is being undermined by the growing number of patients with diabetes mellitus (DM) in the world. Diabetes mellitus, long considered a disease of minor significance to world health, is now emerging as one of the main threats to human health in the $21^{\text {st }}$ century. The past two decades have seen an explosive increase in the number of people diagnosed with diabetes world-wide. In 2011, the International Diabetes Federation (IDF) estimated that about 366 million people worldwide have diabetes mellitus (DM) [5]. Eighty percent (80\%) of these people live in the low and middle-income countries where tuberculosis (TB) is highly prevalent [6].

The liver plays a central and crucial role in the regulation of carbohydrate metabolism and drugs metabolism. Its normal functioning is essential for the maintenance of blood glucose levels and its continuous supply to organs that require glucose as energy source. Liver disease occurring as a consequence of diabetes mellitus includes glycogen deposition, steatosis, non-alcoholic steato-hepatitis (NASH), fibrosis, cirrhosis, biliary disease, cholelithiasis, cholecystitis, and complications of therapy of diabetes or TB or both (cholestatic and necro-inflammatory) [7].

Liver function tests (LFTs) are commonly used in clinical practice to screen for liver disease, monitor the progression of known disease, and monitor the effects of potentially hepatotoxic drugs. Individuals with diabetes have a higher incidence of liver function test abnormalities than those without it. Mild chronic elevations of transaminases often reflect underlying insulin resistance. The most common LFTs include the serum aminotransferases alkaline phosphatase, bilirubin, albumin, and prothrombin time. Aminotransferases, such as alanine amino transferase (ALT) and aspartate aminotransferase (AST), serve as markers of hepatocyte injury. Alkaline phosphatase (ALP), $\gamma$-glutamyl trans-peptidase (GGT), and bilirubin are markers of biliary function and cholestasis. Chronic mild elevation of transaminases is frequently found in type-2 diabetic patients [8]. Diabetes mellitus (DM) is

${ }^{*}$ Corresponding author: Tume B. Christopher, Department of Biochemistry, University of Dschang, Cameroon, Tel: 237699081771; E-mail: tumechrist@yahoo.com

Received: August 07, 2017; Accepted: September 12, 2017; Published September 21, 2017

Citation: Sama LF, Ali IM, Noubom M, Nganou Djinou OI, Wam EC, et al. (2017) Liver Dysfunction in TB-Diabetic and TB Non-Diabetic Patients Admitted in TB Referral Hospital in Western Cameroon. Biochem Anal Biochem 6: 333. doi: 10.4172/2161-1009.1000333

Copyright: (c) 2017 Sama LF, et al. This is an open-access article distributed unde the terms of the Creative Commons Attribution License, which permits unrestricted use, distribution, and reproduction in any medium, provided the original author and source are credited. 
associated with non-alcoholic fatty liver disease (NAFLD), including its severe form, and non-alcoholic steato-hepatitis (NASH). The AntiTubercular Drugs (ATD) frequently causes disturbances in the LFTs in $20 \%$ to $25 \%$ of the patients and clinical hepatitis in $3 \%$ of the cases. Isoniazid and pyrazinamide raise the serum transaminase levels, while Rifampicin causes hyperbilirubinaemia and centrilobular necrosis of the liver [9]. Among patients with diabetes, the risk of chronic liver disease is doubled, independent of alcoholic or viral hepatitis [10]. This study was conducted to examine the variation of LFTs in diabetic patients among TB patients admitted in the Central Referral Hospital, in western Cameroon.

\section{Materials and Methods}

\section{Ethical considerations}

Ethical clearance for this study was obtained from the Ethics Review and Consultancy Committee of the Cameroon Bioethics Initiative (CAMBIN) under the reference number CBI/294/ERCC/CAMBIN on the $16^{\text {th }}$ of September 2014. An authorisation to collect and analyse blood samples was also obtained from the Regional Delegation of Public Health Bamenda and the Baleng Medicalised Centre in Bafoussam. All participants were fully informed of the study goals, procedures, potential harm and benefits, cost as well as the finality of the study. They willingly provided informed consent either by signing or placing their thumbprint on the consent form after being satisfied with responses to all questions asked the investigator. Information was provided in English, French or interpreted in the local dialect by a hospital volunteer independent of the study team.

\section{Study site}

This study was carried out at the TB unit of the Regional Hospital Bamenda (RHB) situated in the Nord-West Region of Cameroon and at the TB unit of the Centre Médical d'Arrondissement (CMA) De Baleng Bafoussam located in the West Region of Cameroon. The two hospitals include more than $80 \%$ of the state's incident TB cases and have dedicated adult pulmonology wards primarily for admission of TB patients during the intensive phase of treatment.

\section{Study design and population}

A cross sectional study was carried out in which patients with a microscopically confirmed diagnosis of pulmonary $\mathrm{TB}$ that were admitted on the TB wards of the Regional Hospital Bamenda (RHB) and CMA Baleng Bafoussam were consecutively recruited during the study period from November 2014 to July 2015. Patients were excluded from the study if they died during the TB diagnosis, initiated TB treatment or died before information regarding diabetes status could be obtained. All the patients who refused informed consent and minors who provided assent but whose guardians/legal representatives refused to consent were also excluded from the study. For each TB case, information about demographics, medical history and cavitary disease status were recorded upon admission to the ward.

\section{Sample size and sampling technique}

A convenient sample of 189 sputum positive pulmonary TB patients who visited the health facilities and who provided consent prior to the onset of any study procedure were included. This sample size was designed according to the prevalence of the co-infection between these two diseases which is $15 \%$ to $25 \%$ [3]. Enrolment of participants was consecutive and based on three sputum smear positivity with Mycobacterium tuberculosis during diagnosis and prior to treatment.

\section{Data Collection Tools}

\section{Questionnaire administration}

A structured questionnaire was administered to all participants to obtain data on socio-demographic characteristics, family history of diabetes, smoking, alcohol consumption, educational level, outdoor activity, and marital status. Those who could not read were assisted to fill the questionnaires. Anthropometric parameters including height and weight were measured by trained nurses following standard procedures. The body mass index $\left(\mathrm{BMI}, \mathrm{kg} / \mathrm{m}^{2}\right.$ ) was calculated by using the formula: BMI=Weight $(\mathrm{kg}) / \mathrm{Height}^{2}\left(\mathrm{~m}^{2}\right)$. Underweight, normal weight, overweight, and obesity were defined using the method described by Wikner et al. [11]. The BMI cut-off value for underweight (severe underweight, moderate underweight, and mild underweight), normal weight, overweight, and obesity were $<18.5 \mathrm{~kg} / \mathrm{m}^{2}\left(<16 \mathrm{~kg} / \mathrm{m}^{2}\right.$, 16 to $16.9 \mathrm{~kg} / \mathrm{m}^{2}$ and 17 to $\left.18.4 \mathrm{~kg} / \mathrm{m}^{2}\right), 18.5$ to $25 \mathrm{~kg} / \mathrm{m}^{2}$ respectively.

\section{Laboratory investigation and specimen collection}

Diagnosis of smear positive pulmonary TB was performed for all patients following the national TB diagnosis guidelines [12]. Briefly, two consecutive sputum samples (first spot and morning-spot) were collected, smeared, and stained with the auramine staining technique. The stained smears were then examined under the oil immersion objective for acid-fast bacilli using the light microscope. Pulmonary TB was confirmed when at least two consecutive smear results were positive for acid fast bacilli assay (AFB) or one sputum specimen was positive with additional X-ray abnormality, as required by the consultant physician. If the smear results were negative with positive clinical symptoms, culture was done using Löwenstein-Jensen media. After admission to the ward, culture was then performed for those who accepted to participate in the study. For those who were TB confirm, fasting blood glucose was performed on capillary blood after an overnight fasting (at least 10 hours without eating or smoking but can drink water during this period). Random blood sugar (RBS) level was measured using the OneTouch Ultra W glucometer from Johnson and Johnson Company, UK.

The diagnosis of DM was based on the recent American Diabetes Association (ADA) guidelines of fasting blood sugar $\geq 126 \mathrm{mg} / \mathrm{dl}$ or a random or casual blood sugar level $\geq 200 \mathrm{mg} / \mathrm{dl}$ in the presence of the classical symptoms of diabetes $[13,14]$ and the hyperglycaemia was noted when the fasting blood glucose was found between 70 to $130 \mathrm{mg} /$ $\mathrm{dL}$ or the postprandial (1 to 2 hours after eating) blood glucose level above $180 \mathrm{mg} / \mathrm{dL}$.

The Oral Glucose Tolerance Test (OGTT) was also perform by giving $75 \mathrm{~g}$ anhydrous glucose dissolved in $200 \mathrm{ml}$ to $300 \mathrm{ml}$ of water to patients to be consumed within 5 minutes, followed by a further 100 $\mathrm{ml}$ of water; and capillary plasma glucose was measured after 2 hours as describe in the W.H.O and ADA guidelines [13,14]. Only those with apparently normal (fasting blood glucose level less than $99 \mathrm{mg} /$ $\mathrm{dL}$ ) glucose levels were subjected to the oral glucose tolerance test and patient were considered diabetic if two successive week's measurement of fasting blood glucose $\geq 126 \mathrm{mg} / \mathrm{dl}$ or 2 h-OGTT $\geq 200 \mathrm{mg} / \mathrm{dl}$. In a patient with classic symptoms of hyperglycaemia or hyperglycaemic crisis, only a random plasma glucose was performed and the patient was considered diabetic if two successive week's random plasma glucose $\geq 200 \mathrm{mg} / \mathrm{dl}[14,15]$. Aspartate aminotransferase (AST), alanine aminotransferase (ALT), alkaline phosphatase (ALP) and gamma glutamyl trasferase (GGT) were estimated by the method recommended by International Federation of Clinical Chemistry (IFCC) and were expressed in International Unit/Liter. 
Citation: Sama LF, Ali IM, Noubom M, Nganou Djinou OI, Wam EC, et al. (2017) Liver Dysfunction in TB-Diabetic and TB Non-Diabetic Patients Admitted in TB Referral Hospital in Western Cameroon. Biochem Anal Biochem 6: 333. doi: 10.4172/2161-1009.1000333

Page 3 of 8

\section{Data management and analysis}

The data was analysed using Statistical Package for Social Sciences (SPSS) Version 18.0. Data were presented as mean \pm SD or as percentages (\%). A p value $<0.05$ was considered to be statistically significant.

\section{Results}

\section{Socio-demographic characteristics of study subjects}

Out of the 189 participants recruited in this study $36.51 \%(69 / 189)$ were females while $63.49 \%(120 / 189)$ were males. The mean age of the participants was $39.05 \pm 14.305$ years with minimal being 12 years and the maximal 82 years. Also, 34.4\% (65/189) of the patients were smokers against $65.6 \%$ (124/189) non-smokers. A total of $73.5 \%$ (139/189) of the participants consumed alcohol against 26.5\% (50/189) non-alcoholic consumers. Also, $40.7 \%(77 / 189)$ had a settled way of life against 59.3\% (112/189). In addition, 32.8\% (62/189) had family history of diabetes against $67.2 \%(127 / 189)$.

Among all the participants, $48.15 \%$ (91/189), 45.50\% (86/189), and $4.23 \%(8 / 189)$ had primary, secondary and higher levels of education, while $57.67 \%$ (109/189) were married, $40.21 \%$ (76/189) unmarried, and $2.12 \%(4 / 189)$ were widows. Furthermore, $38.63 \%$ (73/189) of the total population were HIV positive against 61.37\% (116/189) HIV negative.

Of all the participants, $11.2 \%$ (21/189) were TB-DM, $65.1 \%$

\begin{tabular}{|c|c|c|c|}
\hline \multicolumn{2}{|c|}{ Characteristics } & Effective & Frequency (\%) \\
\hline \multirow{3}{*}{ Sex } & Female & 69 & $36.50 \%$ \\
\hline & Male & 120 & $64.50 \%$ \\
\hline & Total & 189 & $100 \%$ \\
\hline \multirow{5}{*}{ Age (years) } & $\leq 21$ & 27 & $14.30 \%$ \\
\hline & $22-35$ & 80 & $42.30 \%$ \\
\hline & $26-55$ & 71 & $37.60 \%$ \\
\hline & $>55$ & 11 & $5.80 \%$ \\
\hline & Total & 100 & $100 \%$ \\
\hline \multirow{4}{*}{ Marital status } & Married & 109 & $57.67 \%$ \\
\hline & Unmarried & 76 & $40.21 \%$ \\
\hline & Widowed & 4 & $2.12 \%$ \\
\hline & Total & 189 & $100 \%$ \\
\hline \multirow{5}{*}{ Level of education } & None & 4 & $2.10 \%$ \\
\hline & Primary & 91 & $48.15 \%$ \\
\hline & Secondary & 86 & $45.50 \%$ \\
\hline & University & 8 & $4.23 \%$ \\
\hline & Total & 189 & $100 \%$ \\
\hline \multirow{3}{*}{ HIV status } & HIV positive & 73 & $39.70 \%$ \\
\hline & HIV negative & 116 & $60.30 \%$ \\
\hline & Total & 189 & $100 \%$ \\
\hline \multirow{3}{*}{ Alcohol intake } & Yes & 139 & $73.50 \%$ \\
\hline & No & 50 & $26.50 \%$ \\
\hline & Total & 189 & $100 \%$ \\
\hline \multirow{4}{*}{ Smoking status } & Smoker & 65 & $34.40 \%$ \\
\hline & Non smoker & 124 & $65.60 \%$ \\
\hline & Total & 189 & $100 \%$ \\
\hline & Yes & 77 & $40.70 \%$ \\
\hline \multirow{3}{*}{ Settled way of life } & No & 112 & $59.30 \%$ \\
\hline & Total & 189 & $100 \%$ \\
\hline & Yes & 62 & $32.80 \%$ \\
\hline \multirow{2}{*}{$\begin{array}{l}\text { Family history of } \\
\text { diabetes }\end{array}$} & No & 127 & $67.20 \%$ \\
\hline & Total & 189 & $100 \%$ \\
\hline
\end{tabular}

Table 1: General characteristic of the population.

\begin{tabular}{|c|c|c|c|}
\hline \multirow{2}{*}{ Parameters } & \multicolumn{3}{|c|}{ Frequency (\%) } \\
\cline { 2 - 4 } & Normal & Abnormal & Total (\%) \\
\hline GGT & $114(60.3 \%)$ & $75(39.7 \%)$ & $189(100 \%)$ \\
\hline ALAT & $22(11.64 \%)$ & $167(88.36 \%)$ & $189(100 \%)$ \\
\hline ASAT & $16(8.46 \%)$ & $173(91.53 \%)$ & $189(100 \%)$ \\
\hline ALP & $41(21.70 \%)$ & $148(78.3 \%)$ & $189(100 \%)$ \\
\hline
\end{tabular}

GGT: Gamma Glutamyl Transferase; ALP: Alcaline Phosphatase; ALAT: Alanine Amino Transferase ; ASAT: Aspartate Amino Transferase

Table 2: Repartition of the liver profile status in the study population.

(123/189) were TB without DM, 13.2\% (25/189) had impaired oral glucose tolerant test and $10.6 \%(20 / 189)$ had impaired fasting blood glucose.

The mean age of TB-DM was $41.38 \pm 14.36$ years with age found between 21 and 70 years whereas in TB without DM, the mean age was $35.76 \pm 17.64$ with minimum age being 12 years and maximum age 82 years as presented in Table 1.

\section{Repartition of liver profile status in the study population}

Of the 189 study participants, more than half presented abnormal liver function profile with $78.3 \%$ (148/189), 39.7\% (75/189), $88.36 \%$ (167/189), and $91.54 \%(173 / 189)$ presenting abnormal levels of ALP, GGT, ALAT and ASAT respectively as shown in Table 2.

\section{Repartition of the state of liver parameters according to the} characteristics of the population

The repartition of liver profile according to the characteristics of the population showed that, $33.33 \%, 35.77 \%, 56 \%$, and $35 \%$ of TB-DM, TD non-DM, impaired oral glucose tolerant test and impaired fasting blood glucose respectively, had abnormal levels of GGT whereas $80.95 \%, 88.62 \%, 92 \%$, and $90 \%$ of TB-DM, TD non-DM, impaired oral glucose tolerant test and impaired fasting blood glucose respectively, had abnormal levels of ALAT. Also 90.48\%, 59.79\%, 88\%, and 95\% of TB-DM, TD non-DM, impaired oral glucose tolerant test and impaired fasting blood glucose respectively, had abnormal levels of ASAT and $76.19 \%, 77.24 \%, 80 \%$, and $85 \%$ of TB-DM, TD non-DM, impaired oral glucose tolerant test and impaired fasting blood glucose respectively, had abnormal levels of ALP.

This repartition according to sex showed that $57.9 \%$ and $35.3 \%$ male and female participants respectively, had abnormal levels of GGT, whereas $86.0 \%$ and $97.1 \%$ males and females respectively had abnormal levels of ALAT. Also $90.9 \%$ and $94.1 \%$ male and female participants respectively, had abnormal levels of ASAT while $78.5 \%$ and $72.1 \%$ male and female participants respectively, had abnormal levels of ALP.

According to age, participants of age 31-40 years were the most representative (49.2\%) among participants that had abnormal levels of ALP, whereas participants of age 21 to 30 years and 51 to 60 years represented the highest proportion (95.0\%) of participants that had abnormal levels of ALAT. Patients of age 41 to 50 years represented the highest proportion (97.1\%) of participants that had abnormal levels of ASAT and participants of age 21-30 years represented the highest proportion of participants (82.9\%) that had abnormal levels of ALP.

According to HIV serological status, patient with HIV positive status had higher levels of abnormal liver profile with $41.1 \%, 90.41 \%$, 91.78\%, and $82.19 \%$ respectively for GGT, ALAT, ASAT, and ALP compared to HIV negative patients.

In general, most patients consuming alcohol presented abnormal levels of GGT 58 (41.7\%) compared to the non-alcoholic consumers 17 
Citation: Sama LF, Ali IM, Noubom M, Nganou Djinou OI, Wam EC, et al. (2017) Liver Dysfunction in TB-Diabetic and TB Non-Diabetic Patients Admitted in TB Referral Hospital in Western Cameroon. Biochem Anal Biochem 6: 333. doi: 10.4172/2161-1009.1000333

Page 4 of 8

(34.0\%). Most of the patients who had abnormal levels of ALAT were non-alcoholic consumers (94.0\%). Concerning ASAT, the proportion of patients with abnormal levels was the same in alcohol consumers (92.1\%) and non-alcoholic consumers (92.0\%). More so, the majority of the patients having abnormal levels of ALP were alcohol consumers (77.7\%) as shown in Table 3.

\section{Repartition of the means of liver parameters according to the characteristic of the population}

The evaluation of liver parameters according to sex showed that, although the higher values of these parameters ALAT and ALP, there was no significant difference between sex for ASAT $(\mathrm{P}=0.305)$, ALAT

\begin{tabular}{|c|c|c|c|c|c|c|c|c|c|c|c|c|}
\hline \multirow{2}{*}{$\begin{array}{l}\text { Characteris- } \\
\text { tics }\end{array}$} & \multicolumn{2}{|c|}{ GGT } & \multirow{2}{*}{$\begin{array}{c}\text { Total } \\
\mathbf{N}(\%) \mathbf{n}\end{array}$} & \multicolumn{2}{|c|}{ ALAT } & \multirow{2}{*}{$\begin{array}{l}\text { Total } \\
\text { N (\%) } \\
\text { n }\end{array}$} & \multicolumn{2}{|c|}{ ASAT } & \multirow{2}{*}{$\begin{array}{c}\text { Total } \\
\text { N (\%) } \\
\text { n }\end{array}$} & \multicolumn{2}{|c|}{ ALP } & \multirow{2}{*}{$\begin{array}{l}\text { Total } \\
\mathrm{N}(\%) \\
\mathrm{n}\end{array}$} \\
\hline & $\begin{array}{l}\text { Normal } \\
N(\%) n\end{array}$ & $\begin{array}{c}\text { Abnormal } \\
\mathrm{N}(\%) \mathrm{n}\end{array}$ & & $\begin{array}{l}\text { Normal } \\
\mathbf{N}(\%) \mathbf{n}\end{array}$ & $\begin{array}{l}\text { Abnorma } \\
\mathrm{N}(\%) \mathrm{n}\end{array}$ & & $\begin{array}{l}\text { Normal } \\
N(\%) n\end{array}$ & $\begin{array}{l}\text { Abnormal } \\
\mathbf{N}(\%) \mathbf{n}\end{array}$ & & $\begin{array}{l}\text { Normal } \\
\mathbf{N}(\%) \mathbf{n}\end{array}$ & $\begin{array}{c}\text { Abnormal } \\
\text { N (\%) n }\end{array}$ & \\
\hline TB-DM & $\begin{array}{c}14 \\
(66.67 \%)\end{array}$ & $7(33.33 \%)$ & $21(11.11)$ & $4(19.05 \%)$ & 17 (80.95\%) & $\begin{array}{c}21 \\
(11.12 \%)\end{array}$ & $2(9.53 \%)$ & $\begin{array}{c}19 \\
(90.48 \%)\end{array}$ & $\begin{array}{c}21 \\
(11.12 \%)\end{array}$ & $5(23.81)$ & $\begin{array}{c}16 \\
(76.19 \%)\end{array}$ & $\begin{array}{c}21 \\
(11.12 \%)\end{array}$ \\
\hline TB non DM & $\begin{array}{c}79 \\
(64.23 \%)\end{array}$ & $\begin{array}{c}44 \\
(35.77 \%)\end{array}$ & $\begin{array}{c}123 \\
(65.08 \%)\end{array}$ & $\begin{array}{c}14 \\
(11.38 \%)\end{array}$ & $109(88.62 \%)$ & $\begin{array}{c}123 \\
(65.08 \%)\end{array}$ & $\begin{array}{c}10 \\
(8.13 \%)\end{array}$ & $\begin{array}{c}113 \\
(59.79 \%)\end{array}$ & $\begin{array}{c}123 \\
(65.08 \%)\end{array}$ & $28(22.76)$ & $\begin{array}{c}95 \\
(77.24 \%)\end{array}$ & $\begin{array}{c}123 \\
(65.08 \%)\end{array}$ \\
\hline TOGD & $11(44 \%)$ & $14(56 \%)$ & $25(13.23)$ & $2(8 \%)$ & $23(92 \%)$ & $\begin{array}{c}25 \\
(13.23 \%)\end{array}$ & $3(13.63)$ & $22(88 \%)$ & $\begin{array}{c}25 \\
(13.23 \%)\end{array}$ & $5(25 \%)$ & $20(80 \%)$ & $\begin{array}{c}25 \\
(13.23 \%)\end{array}$ \\
\hline GJD & $13(65 \%)$ & $7(35 \%)$ & $20(10.58)$ & $2(11.12 \%)$ & $18(90 \%)$ & $\begin{array}{c}20 \\
(10.58 \%)\end{array}$ & $1(5.26 \%)$ & $19(95 \%)$ & $\begin{array}{c}20 \\
(10.58 \%)\end{array}$ & $3(10.65 \%$ & $17(85 \%)$ & $\begin{array}{c}20 \\
(10.58 \%)\end{array}$ \\
\hline Total & $\begin{array}{c}117 \\
(61.91 \%)\end{array}$ & $72(38.1)$ & $189(100 \%)$ & $\begin{array}{c}22 \\
(11.64 \%)\end{array}$ & $167(88.36 \%)$ & $189(100 \%)$ & $\begin{array}{c}16 \\
(8.47 \%)\end{array}$ & $\begin{array}{c}173 \\
(91.54 \%)\end{array}$ & $189(100 \%)$ & $\begin{array}{c}41 \\
(21.69 \%)\end{array}$ & $\begin{array}{c}148 \\
(78.31 \%)\end{array}$ & $189(100 \%)$ \\
\hline \multicolumn{13}{|c|}{ Sex } \\
\hline Male & $\begin{array}{c}70 \\
(42.1 \%)\end{array}$ & $51(57.9 \%)$ & $121(64.1 \%$ & $17(14.0)$ & $104(86.0 \%)$ & $121(64.1 \%$ & $11(9.1 \%$ & $\begin{array}{c}110 \\
(90.9 \%)\end{array}$ & $121(64.1 \%$ & $26(21.5 \%)$ & 95 (78.5\%) & $121(64.1 \%$ \\
\hline Female & $\begin{array}{c}44 \\
(64.7 \%)\end{array}$ & $24(35.3 \%)$ & $68(35.9 \%$ & $2(2.9 \%)$ & $66(97.1 \%)$ & 68 (35.9\% & $4(5.9 \%)$ & $64(94.1 \%)$ & 68 (35.9\% & $19(27.9 \%)$ & 49 (72.1\%) & $68(35.9 \%$ \\
\hline Total & $\begin{array}{c}114 \\
(60.3 \%)\end{array}$ & 75 (39.7\%) & $189(100 \%)$ & $19(10.1 \%)$ & $170(89.9 \%)$ & $189(100 \%)$ & $15(7.9 \%)$ & $\begin{array}{c}174 \\
(92.1 \%)\end{array}$ & $189(100 \%)$ & $45(23.8 \%)$ & $\begin{array}{c}144 \\
(76.2 \%)\end{array}$ & $100(100 \%)$ \\
\hline \multicolumn{13}{|c|}{ Age stratification (years) } \\
\hline$<21$ & $9(81.8 \%)$ & $2(18.2 \%)$ & $11(5.82 \%)$ & $3(27.3 \%)$ & $8(72.7 \%)$ & $11(5.82 \%)$ & $1(9.1 \%)$ & $10(90.9 \%)$ & $11(5.82 \%)$ & $3(27.3 \%)$ & $8(72.7 \%)$ & $11(5.82 \%)$ \\
\hline $21-30$ & $\begin{array}{c}23 \\
(56.1 \%)\end{array}$ & $18(43.9 \%)$ & $41(21.7 \%)$ & $2(4.9 \%)$ & $39(95.1 \%)$ & $41(21.7 \%)$ & $3(7.3 \%)$ & $38(92.7 \%)$ & $41(21.7 \%)$ & $7(17.1 \%)$ & $34(82.9 \%)$ & $41(21.7 \%)$ \\
\hline $31-40$ & $\begin{array}{c}33 \\
(50.8 \%)\end{array}$ & $32(49.2 \%)$ & $65(34.4 \%)$ & $5(7.7 \%)$ & $60(92.3 \%)$ & $65(34.4 \%)$ & $7(10.8 \%)$ & $58(89.2 \%)$ & $65(34.4 \%)$ & $17(26.2 \%)$ & $48(73.8 \%)$ & $65(34.4 \%)$ \\
\hline $41-50$ & $\begin{array}{c}25 \\
(73.5 \%)\end{array}$ & $9(26.5 \%)$ & $34(17.9 \%)$ & $5(14.7 \%)$ & $29(85.3 \%)$ & $34(17.9 \%)$ & $1(2.9 \%)$ & $33(97.1 \%)$ & $34(17.9 \%)$ & $7(20.6 \%)$ & $27(79.4 \%)$ & $34(17.9 \%)$ \\
\hline $51-60$ & $\begin{array}{c}13 \\
(65.0 \%)\end{array}$ & $7(35.0 \%)$ & $20(10.6 \%)$ & $1(5.0 \%)$ & $19(95.0 \%)$ & $20(10.6 \%)$ & $1(5.0 \%)$ & $19(95.0 \%)$ & $20(10.6 \%)$ & $6(30.0 \%)$ & $14(70.0 \%)$ & $20(10.6 \%)$ \\
\hline $61-70$ & $9(64.3 \%)$ & $5(35.7 \%)$ & $14(7.1 \%$ & $3(21.4 \%)$ & $11(78.6 \%)$ & $14(7.1 \%$ & $2(14.3 \%)$ & $12(85.7 \%)$ & $14(7.1 \%$ & $3(21.4 \%)$ & $11(78.6 \%)$ & $14(7.1 \%$ \\
\hline$>70$ & $2(50.0 \%)$ & $2(50.0 \%)$ & $4(2.2 \%)$ & $0(0.0 \%)$ & $4(100 \%)$ & $4(2.2 \%)$ & $0(0.0 \%)$ & $4(100 \%)$ & $4(2.2 \%)$ & $2(50.0 \%)$ & $2(50.0 \%)$ & $4(2.2 \%)$ \\
\hline \multicolumn{13}{|c|}{ Serological status } \\
\hline $\mathrm{HIV}+$ & $\begin{array}{c}43 \\
(58.9 \%)\end{array}$ & $30(41.1 \%)$ & $\begin{array}{c}73 \\
(38.63 \%)\end{array}$ & $7(9.6 \%)$ & $66(90.41 \%)$ & $\begin{array}{c}73 \\
(38.63 \%))\end{array}$ & $6(8.22 \%)$ & $\begin{array}{c}67 \\
(91.78 \%)\end{array}$ & $\begin{array}{c}73 \\
(38.63 \%))\end{array}$ & $\begin{array}{c}13 \\
(17.80 \%)\end{array}$ & $\begin{array}{c}60 \\
(82.19 \%)\end{array}$ & $\begin{array}{c}73 \\
(38.63 \%))\end{array}$ \\
\hline HIV- & $\begin{array}{c}69 \\
(59.5 \%)\end{array}$ & $45(38.8 \%)$ & $116(61.37)$ & $\begin{array}{c}14 \\
(12.07 \%)\end{array}$ & $\begin{array}{c}102 \\
(87.93 \% \%)\end{array}$ & $116(61.37)$ & $9(7.76 \%)$ & $\begin{array}{c}109 \\
(93.96 \%)\end{array}$ & $116(61.37)$ & $\begin{array}{c}30 \\
(25.86 \%)\end{array}$ & $\begin{array}{c}86 \\
(74.14 \%)\end{array}$ & $116(61.37)$ \\
\hline Total & $\begin{array}{c}112 \\
(59.26 \%)\end{array}$ & 75 (39.68) & $189(100 \%)$ & $\begin{array}{c}21 \\
(11.11 \%)\end{array}$ & $\begin{array}{c}168 \\
(88.89 \% \%)\end{array}$ & $189(100 \%)$ & $15(3.07)$ & $176(93.12)$ & $189(100 \%)$ & $\begin{array}{c}43 \\
(28.75 \%)\end{array}$ & $\begin{array}{c}116 \\
(61.37 \%)\end{array}$ & $189(100 \%)$ \\
\hline \multicolumn{13}{|c|}{ Alcohool intake } \\
\hline Yes & $\begin{array}{c}81 \\
(58.3 \%)\end{array}$ & $58(41.7 \%)$ & $139(73.54)$ & $16(11.5 \%)$ & $123(88.5 \%)$ & $\begin{array}{c}139 \\
(73.55 \%)\end{array}$ & $11(7.9 \%)$ & $\begin{array}{c}128 \\
(92.1 \%)\end{array}$ & $139(73.54)$ & $31(22.3 \%)$ & $\begin{array}{c}108 \\
(77.7 \%)\end{array}$ & $139(73.54)$ \\
\hline No & $\begin{array}{c}33 \\
(66.0 \%)\end{array}$ & $17(34.0 \%)$ & $50(26.46)$ & $3(6.0 \%)$ & $47(94.0 \%)$ & $50(26.46)$ & $4(8.0 \%)$ & $46(92.0 \%)$ & $50(26.46)$ & $14(28.0 \%)$ & $36(72.0 \%)$ & $50(26.46)$ \\
\hline Total & $\begin{array}{c}114 \\
(60.32 \%)\end{array}$ & $\begin{array}{c}75 \\
(36.68 \%\end{array}$ & $189(100 \%)$ & $\begin{array}{c}19 \\
(10.05 \%)\end{array}$ & $170(89.94)$ & $189(100 \%)$ & $15(7.94)$ & $144(76.19)$ & $189(100 \%)$ & $\begin{array}{c}45 \\
(23.81 \%)\end{array}$ & $\begin{array}{c}144 \\
(76.19 \%)\end{array}$ & $189(100 \%)$ \\
\hline \multicolumn{13}{|c|}{ Smoking status } \\
\hline Smoker & $\begin{array}{c}40 \\
(61.5 \%)\end{array}$ & $25(38.5 \%)$ & $65(34.4 \%)$ & $6(9.2 \%)$ & $59(90.8 \%)$ & $65(34.4 \%)$ & $5(7.7 \%)$ & $60(92.3 \%)$ & $65(34.4 \%)$ & $13(20.0 \%)$ & $52(80.0 \%$ & $65(34.4 \%)$ \\
\hline Non smoker & $\begin{array}{c}75 \\
(59.7 \%)\end{array}$ & $50(40.3 \%)$ & $124(65.6 \%$ & $13(10.5 \%)$ & $111(89.5 \%)$ & $124(65.6 \%$ & $10(8.1 \%)$ & $\begin{array}{c}114 \\
(91.9 \%)\end{array}$ & $124(65.6 \%$ & $32(25.8 \%)$ & $92(74.2 \%)$ & $124(65.6 \%$ \\
\hline Total & $\begin{array}{c}115 \\
(60.85)\end{array}$ & $\begin{array}{c}75 \\
(36.68 \%\end{array}$ & $189(100 \%)$ & $\begin{array}{c}19 \\
(10.05 \%)\end{array}$ & $170(89.95)$ & $189(100 \%)$ & $\begin{array}{c}15 \\
(7.93 \%)\end{array}$ & $174(92.07$ & $189(100 \%)$ & $\begin{array}{c}45 \\
(23.80 \%)\end{array}$ & $\begin{array}{c}144 \\
(76.2 \%)\end{array}$ & $189(100 \%)$ \\
\hline \multicolumn{13}{|c|}{ Family history of diabetes } \\
\hline Yes & $36(58.1 \%$ & $26(41.9 \%)$ & $62(32.8 \%)$ & $6(9.7 \%)$ & $56(90.3)$ & $62(32.8 \%)$ & $9(14.5 \%)$ & $53(85.5 \%)$ & $62(32.80)$ & $\begin{array}{c}20 \\
(32.25 \%)\end{array}$ & $\begin{array}{c}42 \\
(67.74 \%)\end{array}$ & $62(32.8 \%)$ \\
\hline No & $\begin{array}{c}74 \\
(58.27 \%)\end{array}$ & $\begin{array}{c}53 \\
(41.73 \%)\end{array}$ & $\begin{array}{c}127 \\
(67.2 \%)\end{array}$ & $12(9.45 \%)$ & $115(90.55 \%)$ & $\begin{array}{c}127 \\
(67.2 \%)\end{array}$ & $5(3.94 \%)$ & $122(96.06)$ & $\begin{array}{c}127 \\
(67.2 \%)\end{array}$ & $\begin{array}{c}29 \\
(22.84 \%)\end{array}$ & $\begin{array}{c}98 \\
(77.16 \%)\end{array}$ & $127(67.20$ \\
\hline
\end{tabular}


Citation: Sama LF, Ali IM, Noubom M, Nganou Djinou OI, Wam EC, et al. (2017) Liver Dysfunction in TB-Diabetic and TB Non-Diabetic Patients Admitted in TB Referral Hospital in Western Cameroon. Biochem Anal Biochem 6: 333. doi: 10.4172/2161-1009.1000333

Page 5 of 8

\begin{tabular}{|c|c|c|c|c|c|c|c|c|c|c|c|c|}
\hline Total & $\begin{array}{c}110 \\
(58.20)\end{array}$ & $79(41.8)$ & $189(100 \%)$ & $18(9.52 \%)$ & $171(90.47 \%)$ & $189(100 \%)$ & $14(7.70)$ & $\begin{array}{c}175 \\
(92.59 \%)\end{array}$ & $189(100 \%)$ & $\begin{array}{c}49 \\
(25.93 \%)\end{array}$ & $\begin{array}{c}140 \\
(74.04 \%)\end{array}$ & $189(100 \%)$ \\
\hline \multicolumn{13}{|c|}{$\begin{array}{c}\text { BMI } \\
\text { Obesity BMI } \geq 30\end{array}$} \\
\hline Males & $0(0.0 \%)$ & $0(0.0 \%)$ & $0(0.0 \%)$ & $0(0.0 \%)$ & $0(0.0 \%$ & $0(0.0 \%)$ & $0(0.0 \%)$ & $0(0.0 \%$ & $0(0.0 \%)$ & $0(0.0 \%)$ & $0(0.0 \%)$ & $0(0.0 \%)$ \\
\hline Females & $4(100 \%)$ & $0(0.0 \%)$ & $4(2.12 \%)$ & $1(25.0 \%)$ & $3(75 \%)$ & $4(2.12 \%)$ & $0(0.0 \%)$ & $4(100 \%)$ & $4(2.12 \%)$ & $2(50.0 \%)$ & $2(50.0 \%)$ & $4(2.12 \%)$ \\
\hline Total & $4(2.12 \%)$ & $0(0.00 \%)$ & $4(2.12 \%)$ & $1(0.53 \%)$ & $3(1.58 \%)$ & $4(2.12 \%)$ & $0(0.0 \%$ & $4(2.12 \%)$ & $4(2.12 \%)$ & $2(1.06 \%)$ & $2(1.06 \%)$ & $4(2.12 \%)$ \\
\hline \multicolumn{13}{|c|}{ Overweight $25 \leq \mathrm{BMI}<30$} \\
\hline Male & $\begin{array}{c}6 \\
(100.0 \%)\end{array}$ & $0(0.0 \%)$ & $6(3.17 \%)$ & $1(16.7 \%)$ & $5(83.3 \%)$ & $6(3.17 \%)$ & $2(33.3 \%)$ & $4(66.7 \%)$ & $6(3.17 \%)$ & $3(50.0 \%)$ & $3(50.0 \%)$ & $6(3.17 \%)$ \\
\hline Female & $1(14.3 \%)$ & $6(85.7 \%)$ & $7(3.70 \%)$ & $0(0.0 \%)$ & $7(100 \%)$ & $7(3.70 \%)$ & $1(14.3 \%)$ & $6(85.7 \%)$ & $7(3.71 \%)$ & $0(0.0 \%)$ & $7(100.0 \%)$ & $7(3.70 \%)$ \\
\hline Total & $7(3.70 \%)$ & $6(3.17 \%)$ & $13(6.89 \%)$ & $1(0.53 \%)$ & $13(6.88 \%)$ & $13(6.88 \%)$ & $3(1.58 \%)$ & $10(5.3 \%)$ & $13(6.88 \%)$ & $3(1.59 \%)$ & $10(5.29 \%)$ & $13(6.88 \%)$ \\
\hline \multicolumn{13}{|c|}{ Normal $18.5 \leq \mathrm{BMI}<25$} \\
\hline Male & $\begin{array}{c}49 \\
(50.5 \%)\end{array}$ & $48(49.5 \%)$ & $\begin{array}{c}97 \\
(51.33 \%)\end{array}$ & $14(14.4 \%)$ & $83(85.6 \%)$ & $\begin{array}{c}97 \\
(51.32 \%)\end{array}$ & $7(7.2 \%)$ & $90(92.8 \%)$ & $\begin{array}{c}97 \\
(51.33 \%)\end{array}$ & $19(19.6 \%)$ & $78(80.4 \%)$ & $\begin{array}{c}97 \\
(51.32 \%)\end{array}$ \\
\hline Female & $\begin{array}{c}26 \\
(66.7 \%)\end{array}$ & $13(33.3 \%)$ & $\begin{array}{c}39 \\
(20.64 \%)\end{array}$ & $0(0.0 \%)$ & $39(100 \%)$ & $\begin{array}{c}39 \\
(20.64 \%)\end{array}$ & $1(2.6 \%)$ & $38(97.4 \%)$ & $39(20.64)$ & $9(23.1 \%)$ & $30(76.9 \%)$ & $\begin{array}{c}39 \\
(20.64 \%)\end{array}$ \\
\hline Total & $\begin{array}{c}75 \\
(39.68 \%)\end{array}$ & $\begin{array}{c}61 \\
(32.26 \%)\end{array}$ & $\begin{array}{c}136 \\
(71.96 \%)\end{array}$ & $14(7.41 \%)$ & $122(64.55 \%)$ & $\begin{array}{c}136 \\
(71.96 \%)\end{array}$ & $8\left(4.23 \%^{\circ}\right.$ & $\begin{array}{c}128 \\
(67.72 \%)\end{array}$ & $\begin{array}{c}136 \\
(71.96 \%)\end{array}$ & $28(14.82)$ & $\begin{array}{c}108 \\
(57.14 \%)\end{array}$ & $\begin{array}{c}136 \\
(71.96 \%)\end{array}$ \\
\hline \multicolumn{13}{|c|}{ Underweight $\mathrm{BMI}<18.5$} \\
\hline Male & $\begin{array}{c}15 \\
(83.3 \%)\end{array}$ & $3(16.7 \%)$ & $18(9.52 \%)$ & $2(11.1 \%)$ & $16(88.9 \%)$ & $18(9.52 \%)$ & $2(11.1 \%)$ & $16(88.9 \%)$ & $18(9.58 \%)$ & $4(22.2 \%)$ & $14(77.8 \%)$ & $18(9.52 \%)$ \\
\hline Female & $\begin{array}{c}13 \\
(72.2 \%)\end{array}$ & $15(27.8 \%)$ & $\begin{array}{c}28 \\
(14.82 \%)\end{array}$ & $1(5.6 \%)$ & $17(94.4 \%)$ & $18(9.52 \%)$ & $2(11.1 \%)$ & $16(88.9 \%)$ & $18(9.58 \%)$ & $8(44.4 \%)$ & $10(55.6 \%)$ & $18(9.52 \%)$ \\
\hline Total & $\begin{array}{c}28 \\
(14.82 \%)\end{array}$ & $18(9.52 \%)$ & $\begin{array}{c}46 \\
(24.86 \%)\end{array}$ & $3(1.58 \%)$ & $33(17.46 \%)$ & $\begin{array}{c}36 \\
(19.05 \%)\end{array}$ & $4(2.12 \%)$ & $\begin{array}{c}32 \\
(16.93 \%)\end{array}$ & $\begin{array}{c}36 \\
(19.05 \%)\end{array}$ & $12(6.35 \%)$ & $24(12.7 \%)$ & $\begin{array}{c}36 \\
(19.05 \%)\end{array}$ \\
\hline
\end{tabular}

Table 3: Repartition of status in the study population.

$(\mathrm{P}=0.934)$ and $\mathrm{GGT}(\mathrm{P}=0.161)$. However, a significant difference was observed for ALP $(\mathrm{P}=0.014)$. Regarding the serological status, high values of ASAT and ALAT were observed although no significant difference was noted between HIV positive and HIV negative patients $(\mathrm{P}=0.538)$ for ASAT, $(\mathrm{P}=0.997)$ for ALAT, $(\mathrm{P}=0.719)$ for $\mathrm{PAL}$ and $(\mathrm{P}=0.314)$ for $\mathrm{GGT}$. Also, no significant difference was observed between age, alcohol intake, smoking status, family history of diabetes and body mass index (BMI). Nonetheless, a significant difference was observed with ALP $(\mathrm{P}=0.021)$ and GGT $(\mathrm{P}=0.022)$ for patients having overweight BMI and also with ALP $(\mathrm{P}=0.000)$ for patients having normal BMI as presented in Table 4.

\section{Repartition of the means of liver profile parameters accord- ing to TB-DM and TB non-DM}

The comparisons of liver profile between TB-DM and TB nonDiabetes showed high levels of liver enzymes between the two types of population but no significant difference was observed between the two populations as shown in Table 5.

Same results were obtained by comparing TB-DM and TB non-DM patients all positive to HIV, but a significant difference was observed with GGT $(\mathrm{P}=0.000)$ as shown in Table 6 . With other characteristics of the population, significant differences were observed within patients presenting impaired oral glucose tolerant test and those presenting impaired fasting blood glucose regarding ALP $(\mathrm{P}=0.013)$ (Table 7).

\section{Repartition of liver profile status according to moderate tox- icity and severe toxicity}

Grouping liver profile into moderate toxicity and severe toxicity between TB-DM and TB non-DM patients are represented in Figures 1 to 4 according to the enzymes. It showed that all patients, TB-DM and TB non-DM had moderate toxicity regarding PAL (Figure 1). Also, $5.3 \%$ of the TB-DM patients presented severe toxicity and no moderate toxicity was observed regarding GGT whereas $7.1 \%$ and $1.2 \%$ of TB non-DM presented moderate and severe toxicity respectively regarding
GGT (Figure 2). As concerns ALAT, $15.80 \%$ and $57.90 \%$ of TB-DM presented moderate and severe toxicities respectively whereas $8.80 \%$ and $70.60 \%$ of TB non-DM respectively presented moderate and severe toxicities (Figure 3). Concerning ASAT, $26.30 \%$ and $57.90 \%$ of TBDM presented moderate and severe toxicities respectively while $20 \%$ and $60 \%$ of TB non-DM respectively presented moderate and severe toxicities regarding ASAT (Figure 4).

\section{Discussion}

Of the 189 study participants, $78.3 \%$ (148/189), 39.7\% (75/189), $88.36 \%$ (167/189), and 91.53\% (173/189) showed abnormal values of ALP, GGT, ALAT, and ASAT respectively. Of these patients with abnormal values, 16 (76.19\%), and 95 (77.24\%); 7 (33.33\%) and 44 (35.77\%); 17(80,95\%) and 109(88,62\%), $19(90.48 \%)$ and $113(59.79 \%)$ of TB-DM and TB non-DM patients respectively for ALP, GGT, ALAT, and ASAT. The most abundant hepatic abnormality with the highest rates was ASAT 91.53\% (173/189), with the highest mean of $551.68 \pm 593.69$ obtained in patients with ages greater than 70 years. The aminotransferases (ALAT and ASAT) levels were high in $88.36 \%(167 / 189)$ and $91.53 \%$ (173/189) of cases, respectively. In the liver, ALAT is only cytoplasmic whereas ASAT is both cytosolic and mitochondrial. Therefore, a serum increase in ALAT levels is more specific in the case of exceptional liver damage caused by alcoholic hepatitis, hepatic cirrhosis and hepatic neoplasia [16].

Other than alcoholic and viral hepatitis, non-alcoholic fatty liver disease is the leading cause of the increase in aminotransferases, which is considered to be the most prevalent disease in type 2 diabetics $[17,18]$.

The activity of Alkaline Phosphatase (ALP) is present in the majority of organs such as, the liver, bones, placenta, intestines and some cancers. Increased serum levels of ALP tend to be observed in extra-hepatic obstruction than intra-hepatic obstruction. Increased hepatic levels may also be observed in patients with primary liver cancer or secondary liver fatty liver disease, and may be observed as a consequence of drug reaction [18]. 
Citation: Sama LF, Ali IM, Noubom M, Nganou Djinou OI, Wam EC, et al. (2017) Liver Dysfunction in TB-Diabetic and TB Non-Diabetic Patients Admitted in TB Referral Hospital in Western Cameroon. Biochem Anal Biochem 6: 333. doi: 10.4172/2161-1009.1000333

Page 6 of 8

\begin{tabular}{|c|c|c|c|c|}
\hline Characteristic & ASAT (U/L) & ALAT (U/L) & ALP (U/L) & GGT (U/L) \\
\hline \multicolumn{5}{|c|}{ Sex } \\
\hline Male & $425.85 \pm 333.52$ & $373.47 \pm 318.91$ & $61.87 \pm 89.17$ & $\begin{array}{c}39.46 \pm \\
104.75\end{array}$ \\
\hline Female & $453.81 \pm 340.94$ & $412.86 \pm 335.15$ & $\begin{array}{c}116.76 \pm \\
120.66\end{array}$ & $\begin{array}{c}53.97 \pm \\
127.46\end{array}$ \\
\hline P.value & 0.305 & 0.934 & 0.014 & 0.161 \\
\hline $95 \% \mathrm{Cl}$ & $-72.56-128.47$ & $-57.73-136.51$ & $24.52-85.27$ & $-19.42-48.41$ \\
\hline \multicolumn{5}{|c|}{ Serological status } \\
\hline VIH- & $427.77 \pm 324.36$ & $380.59 \pm 327.32$ & $\begin{array}{l}86.55 \pm \\
109.84\end{array}$ & $\begin{array}{l}52.54 \pm \\
121.38\end{array}$ \\
\hline $\mathrm{VIH+}$ & $441.26 \pm 344.06$ & $392.28 \pm 324.01$ & $\begin{array}{c}78.38 \pm \\
101.54\end{array}$ & $\begin{array}{c}39.52 \pm \\
107.94\end{array}$ \\
\hline P.value & 0.538 & 0.997 & 0.719 & 0.314 \\
\hline $95 \% \mathrm{Cl}$ & $-85.17-112.16$ & $-83.73-107.12$ & $-38.94-22.59$ & $-46.29-20.25$ \\
\hline \multicolumn{5}{|c|}{ Age stratification (years) } \\
\hline$<21$ & $398.31 \pm 345.21$ & $310.31 \pm 325.07$ & $\begin{array}{l}79.97 \pm \\
115.96\end{array}$ & $30.33 \pm 67.25$ \\
\hline $21-30$ & $491.31 \pm 345.21$ & $475.83 \pm 346.13$ & $\begin{array}{c}79.35 \pm \\
110.64\end{array}$ & $40.26 \pm 49.44$ \\
\hline $31-40$ & $404.55 \pm 357.85$ & $325.12 \pm 269.65$ & $\begin{array}{l}78.98 \pm \\
105.96\end{array}$ & $\begin{array}{c}63.84 \pm \\
171.43\end{array}$ \\
\hline $41-50$ & $518.03 \pm 337.73$ & $398.02 \pm 331.05$ & $61.55 \pm 73.77$ & $\begin{array}{c}41.99 \pm \\
103.59\end{array}$ \\
\hline $51-60$ & $391.56 \pm 233.66$ & $362.25 \pm 260.57$ & $\begin{array}{c}87.74 \pm \\
102.07\end{array}$ & $25.58 \pm 34.15$ \\
\hline $61-70$ & $283.06 \pm 230.39$ & $307.31 \pm 323.07$ & $\begin{array}{c}162.47 \pm \\
155.76\end{array}$ & $27.31 \pm 28.35$ \\
\hline$>70$ & $551.68 \pm 593.69$ & $893.37 \pm 292.08$ & $47.56 \pm 31.81$ & $41.71 \pm 24.12$ \\
\hline P-value & 0.723 & 0.997 & 0.818 & 0.898 \\
\hline \multicolumn{5}{|c|}{ Alcohol intake } \\
\hline Yes & $438.59 \pm 341.73$ & $383.92 \pm 332.31$ & $\begin{array}{l}75.73 \pm \\
100.27\end{array}$ & $\begin{array}{l}50.39 \pm \\
129.87\end{array}$ \\
\hline No & $428.44 \pm 321.05$ & $398.01 \pm 304.79$ & $\begin{array}{c}98.01 \pm \\
115.63\end{array}$ & $28.82 \pm 37.78$ \\
\hline P.value & 1 & 0.419 & 0.417 & 0.064 \\
\hline $95 \% \mathrm{Cl}$ & $-119.61-99.29$ & -211.67 & -68 & -73.67 \\
\hline \multicolumn{5}{|c|}{ Smoking status } \\
\hline Smoker & $429.11 \pm 328.89$ & $379.09 \pm 311.54$ & $70.31 \pm 99.05$ & $37.21 \pm 49.95$ \\
\hline Non smoker & $439.47 \pm 340.28$ & $392.13 \pm 332.26$ & \begin{tabular}{|c|}
$87.56 \pm$ \\
107.46
\end{tabular} & $\begin{array}{l}48.61 \pm \\
135.23\end{array}$ \\
\hline P.value & 0.731 & 0.343 & 0.412 & 0.145 \\
\hline $95 \% \mathrm{Cl}$ & $-91.27-111.98$ & -196.55 & $-14.36-48.87$ & $-22.89-45.67$ \\
\hline \multicolumn{5}{|c|}{ Obesity BMI $\geq \mathbf{3 0}$} \\
\hline Male & $301.22 \pm 300.56$ & $177.63 \pm 220.72$ & $58.58 \pm 51.05$ & $20.99 \pm 3.83$ \\
\hline Female & $301.22 \pm 300.56$ & $177.63 \pm 220.73$ & $58.58 \pm 51.05$ & $20.99 \pm 3.82$ \\
\hline $\mathrm{p}$-value & 1 & 1 & 1 & 1 \\
\hline \multicolumn{5}{|c|}{ Overweight $25 \leq \mathrm{BMI}<30$} \\
\hline Male & $275.92 \pm 346.65$ & $247.92 \pm 143.17$ & $33.77 \pm 25.59$ & $21.73 \pm 10.22$ \\
\hline Female & $636.25 \pm 310.75$ & $421.13 \pm 260.23$ & $19.49 \pm 9.02$ & $53.19 \pm 73.25$ \\
\hline P-value & 0.583 & 0.26 & 0.021 & 0.022 \\
\hline $95 \% \mathrm{Cl}$ & -802.2 & $-90.14-436.56$ & $-36.93-8.37$ & $-35.32-98.24$ \\
\hline \multicolumn{5}{|c|}{ Normal $18.5 \leq \mathrm{BMI}<25$} \\
\hline Male & $451.74 \pm 344.63$ & $395.82 \pm 332.55$ & $41.37 \pm 58.53$ & $\begin{array}{c}44.19 \pm \\
116.35\end{array}$ \\
\hline Female & $477.35 \pm 353.39$ & $449.9 \pm 340.06$ & $\begin{array}{c}132.83 \pm \\
130.09\end{array}$ & $\begin{array}{c}69.93 \pm \\
160.96\end{array}$ \\
\hline P-value & 0.443 & 0.865 & 0 & 0.107 \\
\hline $95 \% \mathrm{Cl}$ & $-104.57-155.79$ & $-71.94-179.08$ & $59.52-123.40$ & $-23.22-74.69$ \\
\hline \multicolumn{5}{|c|}{ Underweight $\mathrm{BM} \mid<18.5$} \\
\hline Male & $336.34 \pm 242.73$ & $294.92 \pm 269.17$ & $\begin{array}{c}181.73 \pm \\
136.78\end{array}$ & $19.95 \pm 18.34$ \\
\hline Female & $365.75 \pm 316.67$ & $382.76 \pm 366.92$ & $\begin{array}{c}132.72 \pm \\
116.19\end{array}$ & $27.01 \pm 51.08$ \\
\hline
\end{tabular}

\begin{tabular}{|c|c|c|c|c|}
\hline P-value & 0.123 & 0.285 & 0.423 & 0.207 \\
\hline $95 \% \mathrm{Cl}$ & $-161.71-220.53$ & -435.96 & $\begin{array}{c}-134.98- \\
33.06\end{array}$ & -55.89 \\
\hline \multicolumn{5}{|c|}{ Family history of diabetes } \\
\hline Yes & $421.69 \pm 354.35$ & $357.79 \pm 303.92$ & $85.47 \pm 97.76$ & $40.28 \pm 85.99$ \\
\hline No & $430.13 \pm 308.84$ & $403.57 \pm 327.04$ & $\begin{array}{c}82.12 \pm \\
109.78\end{array}$ & $\begin{array}{c}47.22 \pm \\
127.08\end{array}$ \\
\hline P-Value & 0.071 & 0.327 & 0.306 & 0.703 \\
\hline $95 \% \mathrm{Cl}$ & $-91.52-108.38$ & $-52.54-144.09$ & $-35.94-29.25$ & $-28.44-42.32$ \\
\hline \multicolumn{5}{|c|}{ Settled way of life } \\
\hline Yes & $439.47 \pm 341.71$ & $394.99 \pm 326.86$ & $\begin{array}{l}86.21 \pm \\
108.01\end{array}$ & $35.85 \pm 69.96$ \\
\hline No & $430.73 \pm 328.58$ & $376.95 \pm 322.91$ & $\begin{array}{l}74.96 \pm \\
100.02\end{array}$ & $\begin{array}{c}57.53 \pm \\
156.01\end{array}$ \\
\hline P-Value & 0.723 & 0.824 & 0.537 & 0.032 \\
\hline $95 \% \mathrm{Cl}$ & $-106.99-89.53$ & -189.976 & $-41.86-19.37$ & $-11.36-54.72$ \\
\hline
\end{tabular}

GGT: Gamma Glutamyl Transferase; ALP: Alcaline Phosphatase; ALAT: Alanine Amino Transferase ; ASAT: Aspartate Amino Transferase

Table 4: Repartition of the means of liver parameters according to the characteristic of the population.

\begin{tabular}{|c|c|c|c|}
\hline Tests & $\begin{array}{c}\text { TB-DM (mean } \mathbf{\pm} \text { SD) } \\
\mathbf{( n = 2 1 )}\end{array}$ & $\begin{array}{c}\text { TB Non-DM (mean } \mathbf{\pm} \text { SD) } \\
\mathbf{( n = 1 2 3 )}\end{array}$ & P-value \\
\hline ASAT & $381.45 \pm 261.29$ & $441.99 \pm 342.96$ & 0.105 \\
\hline ALAT & $309.11 \pm 304.45$ & $396.42 \pm 326.35$ & 0.69 \\
\hline ALP & $103.38 \pm 137.58$ & $79.19 \pm 100.59$ & 0.194 \\
\hline GGT & $67.98 \pm 183.54$ & $42.08 \pm 103.11$ & 0.094 \\
\hline
\end{tabular}

Table 5: Repartition of liver profile parameters according to TB-DM and TB non diabetes.

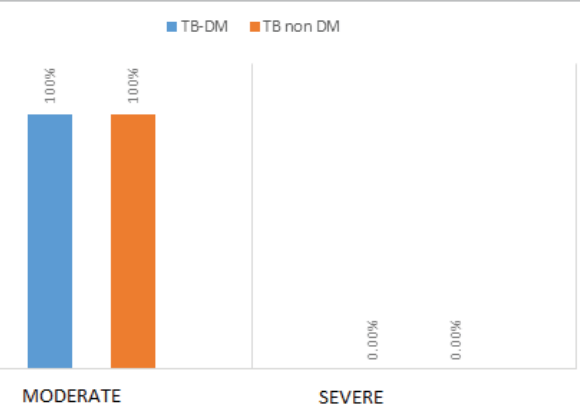

Figure 1: Distribution of patients according to moderate and severe toxicity of ALP.

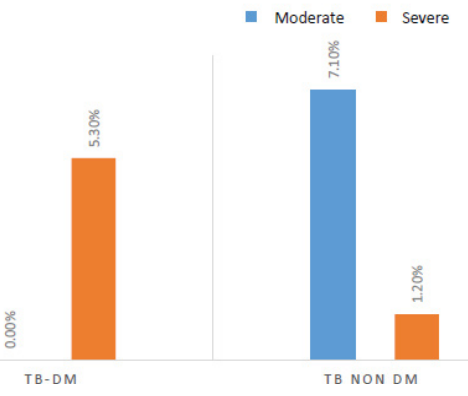

Figure 2: Distribution of patients according to moderate and severe toxicity of GGT.

Gamma-glutamyltransferase (GGT) is present in the liver. It is more sensitive than ALP, transaminases and Leucine aminopeptidase (LAP) in the detection of jaundice, cholangitis and cholecystitis. High GGT levels increase in early stages of liver disease such as infectious hepatitis, but high GGT levels have been observed in chronic alcoholism, diabetes, and some neurological disorders [18]. 
Comparison of the values of liver parameters by sex revealed that the rates of these parameters were higher in female patients than in male patients although the number of male patients with abnormal levels of these liver parameters was higher than that of the females. The difference observed was not equally significant between HIV positive and HIV negative patients. This may be justified by female hormonal factors and also by the fact that HIV positive patients were more of females than males. In addition, HIV has been shown to attack cells directly [19], causing cell death and releasing the cellular content of $20 \%$ of the enzymes [20] and this may be responsible for the increase in liver enzymes in HIV infected patients.

We also observed differences although not significant between age groups with higher rates in older patients. These findings corroborate with a study in Addis Ababa, Ethiopia where a significant correlation

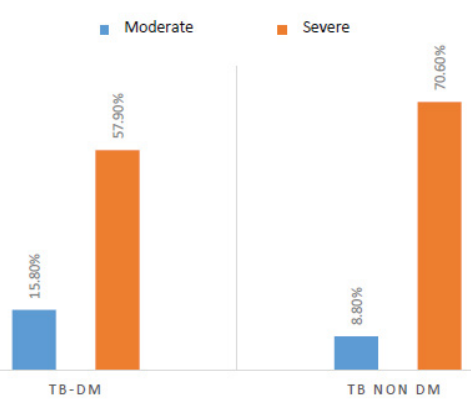

Figure 3: Distribution of patients according to moderate and severe toxicity of ALAT.

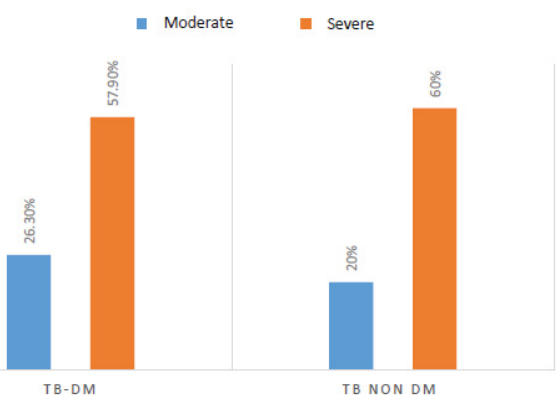

Figure 4: Distribution of patients according to moderate and severe toxicity of ASAT

\begin{tabular}{|c|c|c|c|}
\hline Tests & $\begin{array}{c}\text { TB-DM-HIV+ } \\
\text { (mean } \pm \text { SD) } \mathbf{( N = 1 2 )}\end{array}$ & $\begin{array}{c}\text { TB Non-DM-HIV+ } \\
\text { (means } \pm \mathbf{~ S D ) ~} \mathbf{( N = 4 1 )}\end{array}$ & P value \\
\hline ASAT & $448.87 \pm 243.03$ & $425.59 \pm 332.96$ & 0.238 \\
\hline ALAT & $287.87 \pm 225.78$ & $390.13 \pm 335.83$ & 0.101 \\
\hline ALP & $52.58 \pm 44.28$ & $90.05 \pm 114.09$ & 0.106 \\
\hline GGT & $143.49 \pm 298.95$ & $43.17 \pm 85.54$ & 0 \\
\hline
\end{tabular}

Table 6: Repartition of liver profile parameters according to TB-DM and TB nondiabetes positive to HIV.

\begin{tabular}{|c|c|c|c|c|}
\hline Characteristic & ASAT (U/L) & ALAT (U/L) & ALP (U/L) & GGT (U/L) \\
\hline Impaired oral glucose & $335.97 \pm$ & $395.09 \pm$ & $131.48 \pm$ & $87.81 \pm$ \\
tolerance & 289.64 & 315.05 & 137.88 & 216.90 \\
\hline Impaired fasting blood & $435.91 \pm$ & $387.64 \pm$ & $81.63 \pm$ & $44.68 \pm$ \\
sugar & 335.57 & 324.51 & 104.70 & 113.33 \\
\hline P.value & 0.164 & 0.541 & 0.013 & 0.052 \\
\hline $95 \% \mathrm{Cl}$ & $-218.98-$ & $-267.84-$ & $-75.79-$ & -175.6 \\
\hline
\end{tabular}

Table 7: Repartition of liver profile parameters according to other characteristics of the population. was obtained between age and hepatic enzymes [21] in diabetic patients and a control population.

In addition, we obtained differences although not significant, of the liver parameters between the smoking and non-smoking patients and also between the patients who consume alcohol and those who do not consume alcohol. However, it has been shown that alcohol consumption leads to increased liver enzymes [16]. This non-significant difference may be due to the fact that patients who consumed alcohol had long stopped drinking alcohol at the onset of the disease, especially during self-medication (over-the-counter drugs).

The assessment of hepatic parameters based on body mass index (which is gender-specific) revealed that the differences observed accurately with ALT and ASAT were not significant. This result does not corroborate with those obtained by Subir et al. [21]. This difference can be explained by the difference in the study populations. On the other hand, a significant difference was observed with ALP and GGT in overweight patients and also with ALP in patients with normal BMI.

The assessment of hepatic parameters in TB-DM and TB non-DM patients revealed differences between patients in these two population groups although these differences were not significant. These results do not corroborate with those obtained in India and Addis Ababa, Ethiopia where significant differences were observed between diabetic patients and the control population (non-diabetic), $[21,16]$. This difference can be explained not only by the difference between the study populations but also with the fact that patients in their study populations were on anti-diabetic treatment for a long period of time.

In general, the values of the parameters were very high in our study population with patients presenting moderate toxicity (liver profile $>2.5$ times normal values) and also severe toxicity (hepatic profile $>5.1$ times normal values). This could be explained by the high prevalence of HIV positive patients (39.7\%) in our study population. It has been shown that the liver is an important site of HIV replication causing the death of liver cells and increasing the levels of liver parameters [20]. High levels of liver parameters have been observed in HIV patients, and these rates are much higher in the case of tuberculosis infection [22]. This may be due to connective tissue degeneration of the liver and also to hepatobiliary obstruction caused by tuberculosis infection in these patients.

These high rates of liver function parameters may also be attribbuted to the high prevalence of viral hepatitis B and C $(12.7 \%$ and $4.23 \%$ respectively) in this study population [23]. It has been demonstrated that viral hepatitis $\mathrm{B}$ and $\mathrm{C}$ are independently and significantly associated with the incidence of hepatotoxicity [24] and antiretroviral therapy [25].

This can also be explained by the fact that access to treatment in our society is still difficult because of poverty. Also, the majority of our study population generally take unprescribed medications of unknown origins, usually poorly preserved and in most cases expired and consequently toxic and visit hospitals for appropriate treatment only if their health conditions become critical [23].

\section{Conclusion}

This study showed high levels of liver enzymes in TB-diabetic and TB non-diabetic patients but no significant difference was observed between the two populations. Therefore, proper follow-up during TB treatment should be mandatory.

\section{Acknowledgements}

We wish to thank all the participants who sacrificed their time and donated their 
Citation: Sama LF, Ali IM, Noubom M, Nganou Djinou OI, Wam EC, et al. (2017) Liver Dysfunction in TB-Diabetic and TB Non-Diabetic Patients Admitted in TB Referral Hospital in Western Cameroon. Biochem Anal Biochem 6: 333. doi: 10.4172/2161-1009.1000333

Page 8 of 8

blood for this study. We also thank particularly the Director, and Nurses of Regional Hospital Bamenda and "Centre Médical d'Arrondissement de Bafoussam" for their contributions and assistance during sample collection and analysis.

\section{References}

1. Anthea M, Hopkins J, McLaughlin CW, Johnson S, Warner MQ, et al. (1993) Human biology and health. Englewood Cliffs, Prentice Hall Science, New Jersey, USA. pp-256.

2. Dye C (2006) Global epidemiology of tuberculosis. The Lancet 367: 938-940.

3. Blanca IR, Aulasa JC, Mohammad HR, Weiwei W, Mary AR, et al. (2011) Cross-sectional assessment reveals high diabetes prevalence among newlydiagnosed tuberculosis cases. Bull World Hlth Org 89: 352-359.

4. WHO (2010) Global Tuberculosis Control: WHO Report 2010, Geneva, Switzerland.

5. Kibirige D, Richard S, Edrisa M, William W (2013) Overt diabetes mellitus among newly diagnosed Ugandan tuberculosis patients: A cross sectional study. BMC Infec Dis 13: 122.

6. International Diabetes Federation (2011) IDF Diabetes Atlas, Belgium.

7. Gavin NL, Tavill AS (1999) Liver disease and diabetes mellitus. Clin Diab 17: 85.

8. Elizabeth $\mathrm{HH}$ (2005) Elevated liver function tests in type 2 diabetes. Clin Diab 23: $115-119$

9. Ragni MV, Belle SH, Im K, Neff G, Roland M, et al. (2003) Survival of human immune deficiency virus-infected liver transplant recipients. J Infect Dis 188: $1412-1420$.

10. West J, Brousil J, Gazis A, Jackson P, Mansell A, et al. (2004) Elevated serum alanine transaminase in patients with type 1 or type 2 diabetes mellitus. Endocr Rev 227: 20-29.

11. Wikner C, Gigante B, Helle-nius ML, De Faire U, Leander K (2013) The risk of type 2 diabetes in men is synergistically affected by parental history of diabetes and overweight. PLoS ONE 8: e61763.

12. WHO (2006) Definition and diagnosis of diabetes mellitus and intermediate hyperglycemia. Report of a WHO/IDF consultation, Geneva, Switzerland.

13. American Diabetes Association (2006) Diagnosis and classification of diabetes mellitus. Diabetes Care 29: S43-S48.

14. American Diabetes Association (2012) Standards of medical care in diabetes. Diabetes Care 35: S11-S63.

15. WHO/IDF (2006) Definition and diagnosis of diabetes mellitus and intermediate hyperglycemia, Geneva, Switzerland.

16. Roshan T, Thanpari C, Paras TS (2014) Liver dysfunction in diabetic patients admitted in referral hospital. Bali Medical J 3: 122-124

17. Joe V, Molly J, Robert KM (2012) Clinical biochemistry. Harper's Illustrated Biochemistry. pp: 721.

18. Mauro P, Renze B (2008) Enzymes. Tietz Fundamentals of Clinical Chemistry. pp: 323-326

19. Oluwafemi O, Oguntibeju B, Olatubosan B (2003) A study on the activities of liver enzymes in HIVIAIDS patients. J Med Sci 3: 106-109.

20. Wild-Up M, Fortuin F, Whittle DHC, Hall AJ, Wolf CR, et al. (1990) Liver and hepatitis B virus in Gambian children. Cancer Epidemiol Biomarkers Prev 2 : 555-561.

21. Belay Z, Daniel S, Tedla K, Gnanasekaran N (2014) Impairment of liver function tests and lipid profiles in type 2 diabetic patients treated at the diabetic center in Tikur Anbessa specialized Teaching Hospital (Tasth), Addis Ababa, Ethiopia. J Diabetes Metab 5: 454

22. Subir KD, Indranath G, Debojyoti B, Praveen A, Sumanta J, et al. (2013) Liver function profile anomalies in HIV seropositive tuberculosis. J Clin Diagn Res 7: 1068-1072.

23. Sama LF, Djinou OIN, Wam EC, Bamou R, Ali IM, et al. (2017) Sero-prevalence of Hepatitis $B$ and $C$ virus and high risk of hepatotoxicity among TB/HIV positive and HIV negative population in Western Cameroon. Glob J Infect Dis Clin Res 3: 001-008.

24. Lomtadze N, Kupreishvili L, Salakaia A, Vashakidze S, Sharvadze L, et al. (2013) Hepatitis C virus co-infection increases the risk of anti-tuberculosis drug-induced hepatotoxicity among patients with pulmonary tuberculosis. PLoS ONE 8: e83892.

25. Ayelagbe OG, Akerele OP, Onuegbu AJ, Oparinde DP (2014) Drug hepatotoxicity in HIV patients on Highly Active Antiretroviral Therapy [HAART] in Southwest Nigeria. J Dental Medical Sci 13: 67-70. 